\title{
Erratum: Direct detection limits on heavy dark matter [Phys. Rev. D 102, 123026 (2020)]
}

\author{
Michael Clark®, Amanda Depoian, Bahaa Elshimy, Abigail Kopec, Rafael F. Lang, and Juehang Qin
}

(Received 6 December 2021; published 29 December 2021)

DOI: $10.1103 /$ PhysRevD.104.129903

A numerical error was found in our code, which did not properly account for the Helm form factor when calculating the MIMP-nucleon cross section. That error, together with a counteracting unit error on the dark matter cross section, had extended our XENON1T limit towards higher MIMP masses near the $10^{18} \mathrm{GeV} / \mathrm{c}^{2}$ flux limit given in Ref. [1]. This contradicted the expected multiple-scatter signature above the cross section of $10^{-33} \mathrm{~cm}^{2}$ in the same reference.

We correct for these errors in both of our MAJORANA and XENON1T exclusion regions, resulting in shifted exclusion limits by MAJORANA, weaker exclusion limits by XENON1T at high MIMP mass, and a lower upper bound of its single scattering exclusion region. This weaker limit makes a dedicated multiscatter analysis in XENON1T, and similar liquid xenon detectors more desirable. At this occasion, we also implemented a better detection efficiency model derived from Fig. 1 of Ref. [2] instead of our previous hard $10 \mathrm{keV}_{n r}$ detection threshold, although this only has minor impact on the final exclusion regions. In Sec. II of the original paper, ".. deposit an energy above $1 \mathrm{MeV}$ in the muon veto, which corresponds to particles with cross sections $>10^{-25} \mathrm{~cm}^{2}$ " should be corrected to $10^{-28} \mathrm{~cm}^{2}$. In Sec. III, the text " $\ldots$ the mean free path of a MIMP becomes equal to the size of the detector $(\sim 1 \mathrm{~m})$ once the cross section exceeds $10^{-29} \mathrm{~cm}^{2}$." should state $10^{-32} \mathrm{~cm}^{2}$ instead. Here, we show the revised exclusion regions in Figs 1-3 as updated versions of Figs. 2-4 from the original paper.

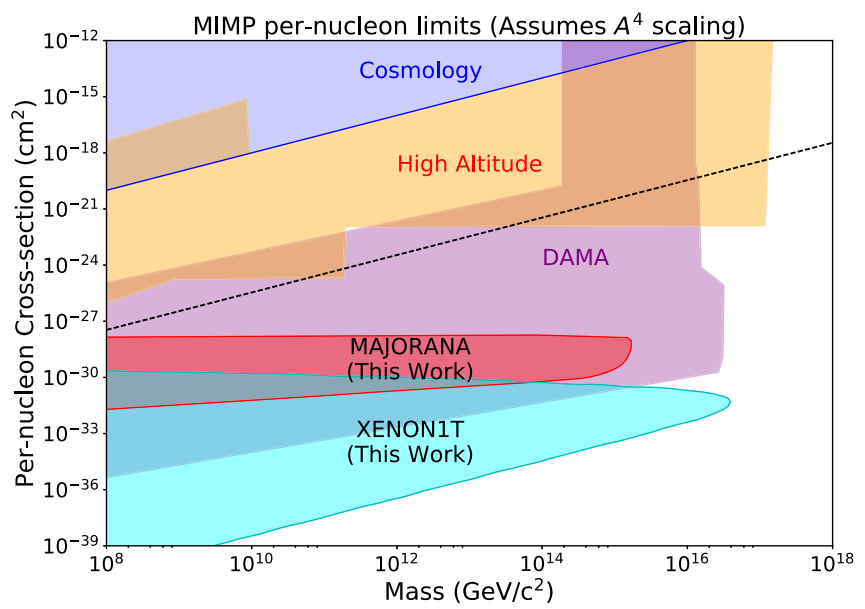

FIG. 1. Corrected version of Fig. 2. Excluded regions in the parameter space of MIMP-nucleon cross section vs MIMP mass from various sources, as well as the limits calculated here from the Majorana Demonstrator (red) and XENON1T (cyan). The dashed black line shows the maximum sensitivity for underground detectors due to the overburden of the Earth's crust. $\mathrm{A}^{4}$ scaling is assumed due to coherent interactions with the nucleus. The correction made in this erratum shifts both MAJORANA and XENON1T limits towards lower cross sections and a weaker MIMP mass limit for XENON1T.

Published by the American Physical Society under the terms of the Creative Commons Attribution 4.0 International license. Further distribution of this work must maintain attribution to the author(s) and the published articles title, journal citation, and DOI. 


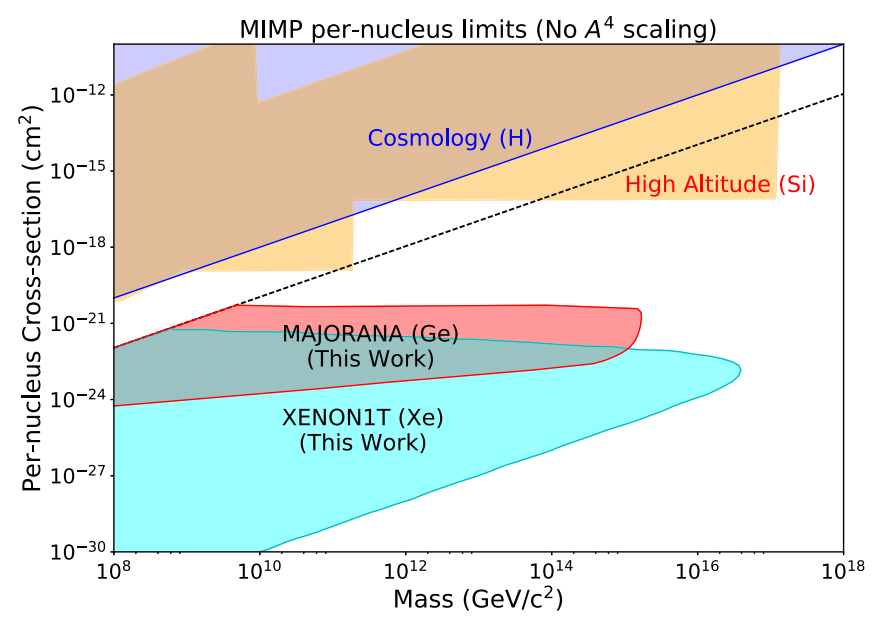

FIG. 2. Corrected version of Fig. 3. Excluded regions in the parameter space of MIMP-nucleus cross section vs MIMP mass cited in the original paper, as well as the corrected limits for the Majorana Demonstrator (red) and XENON1T (cyan). The corresponding nucleus used to correct for the $\mathrm{A}^{4}$ scaling is shown in the labels of each region. The correction made in this erratum shifts both MAJORANA and XENON1T limits towards lower cross sections and a weaker MIMP mass limit for XENON1T.

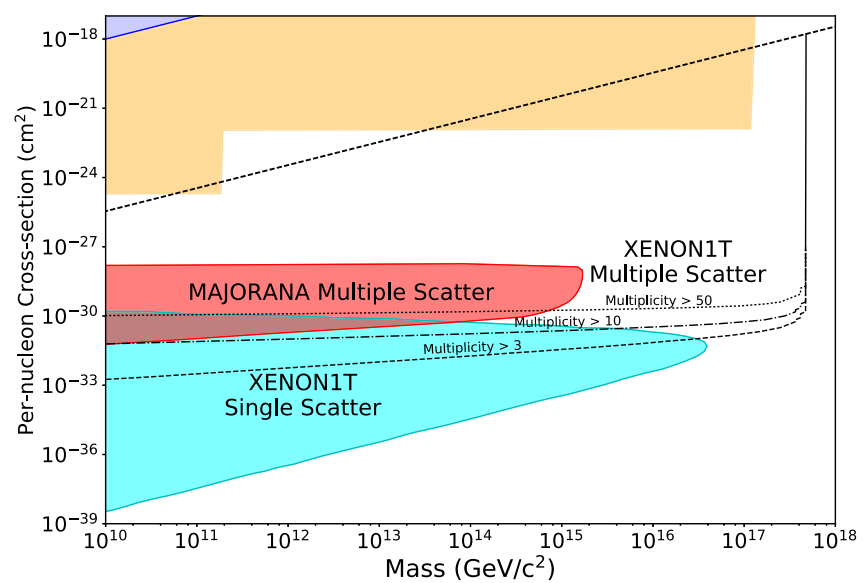

FIG. 3. Corrected version of Fig. 4. The possible sensitivity curves for a dedicated MIMP analysis of the XENON1T dark matter search data (assuming $\mathrm{A}^{4}$ scaling), considering events with scattering multiplicities greater than 3 (dashed), greater than 10 (dashed dotted), and greater than 50 (dotted). The corrected 3, 10, and 50 multiplicity lines have smaller gaps, and the "Multiplicity $>50$ " line is shifted down by 1.5 orders of magnitude. The impact on the vertical "flux limit" is negligible.

We are grateful to Dr. Shengchao Li of Purdue University for the preparation of this erratum and implementing the described improvements. We are also grateful to Nirmal Raj, Ibles Samblas, and Shawn Westerdale for useful discussions.

[1] J. Bramante, B. Broerman, R. F. Lang, and N. Raj, Saturated overburden scattering and the multiscatter frontier: Discovering dark matter at the Planck mass and beyond, Phys. Rev. D 98, 083516 (2018).

[2] E. Aprile et al. (XENON Collaboration), Dark Matter Search Results from a One Ton-Year Exposure of XENON1T, Phys. Rev. Lett. 121, 111302 (2018). 